\title{
THE UNIQUENESS OF MULTIPLICATION IN FUNCTION ALGEBRAS
}

\author{
KRZYSZTOF JAROSZ
}

\begin{abstract}
Let $A$ be a function algebra. We prove that the original multiplication of $A$ is a unique multiplication on the underlying Banach space of $A$ which produces a Banach algebra with the same unit as the original one.
\end{abstract}

Let $A$ be a complex Banach algebra with unit. We denote by 1 the unit of $A$, the norm by $\|\cdot\|$ and the product of $f$ and $g$ by $f g$ or $f \cdot g$. By the definition of a Banach algebra, for any elements $f, g$ in $A$ we have

$$
\|f \cdot g\| \leqslant\|f\|\|g\|
$$

and

$$
\mathbf{1} \cdot f=f .
$$

Suppose now that $A$ is uniform algebra, that is, $A$ is a commutative Banach algebra with unit and $\left\|f^{2}\right\|=\|f\|^{2}$ for all $f$ in $A$. Our goal is to prove that there exists exactly one (associative) multiplication on the Banach space $A$ which satisfies (1) and (2). This result follows upon considering a more general situation.

By an $\varepsilon$-deformation of $A$ we mean an associative multiplication $\times$ on the Banach space $A$ such that

$$
\|f \times g-f \cdot g\| \leqslant \varepsilon\|f\|\|g\| \text { for all } f, g \text { in } A .
$$

This definition was formulated by Johnson [2] (see also [3,4]). He investigates whether all multiplications on a Banach algebra $A$ near the given multiplication share some of the properties of the original one. Small deformations of function algebras were studied deeply by $\mathbf{R}$. Rochberg [5].

If $X$ is an $\varepsilon$-deformation of the multiplication of a Banach algebra $A$ then for all $f, g$ in $A$

$$
\|f \times g\| \leqslant(1+\varepsilon)\|f\|\|g\|
$$

and

$$
\|\mathbf{1} \times f-f\| \leqslant \varepsilon\|f\| .
$$

Our main theorem shows that for uniform algebras the converse implication also holds.

Received by the editors October 5, 1982.

1980 Mathematics Subject Classification. Primary 46J10; Secondary 46J35.

Key words and phrases. Function algebras, perturbations of multiplication, Nagasawa's Theorem, uniqueness of multiplication. 
Theorem 1. Suppose $(A, \cdot)$ is a complex uniform algebra. There are positive constants $\varepsilon_{0}, c$ which do not depend on $A$ such that for any $0 \leqslant \varepsilon \leqslant \varepsilon_{0}$ and any multiplication with unit on $A$ satisfying the conditions

(i) $\|f \times g\| \leqslant(1+\varepsilon)\|f\|\|g\|$ and

(ii) $\|\mathbf{1} \times f-f\| \leqslant \varepsilon\|f\|$ for all $f$, $g$ in $A$, we have

(iii) $\|f \times g-f \cdot g\| \leqslant c \sqrt{\varepsilon}\|f\|\|g\|$ for all $f, g$ in A. Moreover the new multiplication $X$ is commutative.

Proof. If $\varepsilon_{0}<1$ then the condition (ii) implies that the operator $T: A \rightarrow A$ : $f \mapsto \mathbf{1} \times f$ is an isomorphism so there exists an element $e$ of $A$ such that $\mathbf{1} \times e=\mathbf{1}$. It is easy to check that $e$ is the unit of the algebra $(A, \times)$ and that the element 1 is invertible in this algebra. A simple computation using (i) and (ii) proves that

$$
\left\|f \times g-1^{-1} \times f \times g\right\| \leqslant \frac{2(1+\varepsilon)^{2} \varepsilon}{1-\varepsilon}\|f\|\|g\|
$$

for all $f, g$ in $A$. Hence the multiplication $\hat{\times}$ defined by $f \hat{\times} g=\mathbf{1}^{-1} \times f \times g$ has the same unit as the original multiplication of the function algebra $A$ and the multiplication denoted by $\hat{X}$ is a $k \varepsilon$-deformation of the multiplication $\times$. This proves that without loss of generality we may assume that the element $\mathbf{1}$ is a common unit of both multiplications $x$ and .

Let us now introduce some notation.

By $\partial A$ and $\mathrm{Ch} A$ we denote the Shilov and the Choquet boundaries of $A$, respectively. Let

$$
\begin{aligned}
\Omega= & \left\{x+i y \in \mathbf{C}:\left(x-\frac{1}{2}\right)^{2}+\left(y-\frac{1}{2}\right)^{2}<\frac{1}{2}\right\} \\
& \cap\left\{x+i y \in \mathbf{C}:\left(x-\frac{1}{2}\right)^{2}+\left(y+\frac{1}{2}\right)^{2}<\frac{1}{2}\right\}
\end{aligned}
$$

and for $r>0$

$$
D(r)=\left\{x+i y \in \mathbf{C}: x^{2}+y^{2}<r^{2}\right\} .
$$

Notice that without loss of generality we may assume that $A$ is an algebra of continuous functions on $\partial A$.

Fix $\delta>0$, and let $\kappa: \overline{D(1)} \rightarrow \bar{\Omega}$ be a continuous map of $\overline{D(1)}$ onto $\bar{\Omega}$ such that $\kappa$ is analytic on $\overline{D(1)}$ and

$$
\kappa(1)=1 \text { and } \kappa(0)=\delta / 2 \text {. }
$$

Let $V \subset \mathbf{C}$ be a neighborhood of 0 such that

$$
\kappa(V) \subset \bar{\Omega} \cap D(\delta) .
$$

Now fix any point $s_{0} \in \mathrm{Ch} A$ and any of its neighborhoods $U \subset \partial A$, and let $f \in A$ be such that

$$
\|f\|=f\left(s_{0}\right)=1 \text { and } f(\partial A-U) \subset V .
$$

The function $\kappa \circ f \in A$ has the following properties:
(a) $\kappa \circ f(\partial A) \subset \bar{\Omega}$;
(b) $\|\kappa \circ f\|=\kappa \circ f\left(s_{0}\right)=1$;
(c) $\kappa \circ f(\partial A-U) \subset \bar{\Omega} \cap D(\delta)$. 
Hence for any $s_{0} \in \mathrm{Ch} A$ there exists a net $\left(f_{\alpha}\right) \subset A$ such that

(A) $f_{\alpha}(\partial A) \subset \bar{\Omega}$,

(B) $\left\|f_{\alpha}\right\|=f_{\alpha}\left(s_{0}\right)=1$,

(C) $\left(f_{\alpha}\right)$ tends uniformly to zero on the compact subsets of the set $\partial A-\left\{s_{0}\right\}$.

Using the net $\left(f_{\alpha}\right)$ we define

$$
g_{\alpha}^{\prime}=f_{\alpha}+i\left(\mathbf{1}-f_{\alpha}\right), \quad g_{\alpha}^{\prime \prime}=f_{\alpha}-i\left(\mathbf{1}-f_{\alpha}\right) .
$$

By direct computation

$$
g_{\alpha}^{\prime} \times g_{\beta}^{\prime}=f_{\alpha}+f_{\beta}-\mathbf{1}+i\left(f_{\alpha}+f_{\beta}-2 f_{\alpha} \times f_{\beta}\right) .
$$

Further observe that, by the definition of $\Omega$, we have

$$
\left\|g_{\alpha}^{\prime}\right\|=\sup _{s \in \partial A}\left|f_{\alpha}(s)+i\left(1-f_{\alpha}(s)\right)\right| \leqslant \sup _{z \in \Omega}|z+i(1-z)|=1 .
$$

Hence from (i) we get

$$
1+\varepsilon \geqslant\left\|g_{\alpha}^{\prime} \times g_{\beta}^{\prime}\right\| \geqslant\left|g_{\alpha}^{\prime} \times g_{\beta}^{\prime}\left(s_{0}\right)\right|=\left|1+2 i\left(1-f_{\alpha} \times f_{\beta}\left(s_{0}\right)\right)\right| \text {. }
$$

The same computations for the functions $g_{\alpha}^{\prime \prime}$ and $g_{\beta}^{\prime \prime}$ show that

$$
1+\varepsilon \geqslant\left\|g_{\alpha}^{\prime \prime} \times g_{\beta}^{\prime \prime}\right\| \geqslant\left|g_{\alpha}^{\prime \prime} \times g_{\beta}^{\prime \prime}\left(s_{0}\right)\right|=\left|1-2 i\left(1-f_{\alpha} \times f_{\beta}\left(s_{0}\right)\right)\right| .
$$

Inequalities (6) and (7) can be satisfied simultaneously only if

$$
\left|1-f_{\alpha} \times f_{\beta}\left(s_{0}\right)\right| \leqslant \sqrt{\varepsilon / 2+\varepsilon^{2} / 4} \leqslant \sqrt{\varepsilon} .
$$

Now for any $g \in A$ define two functionals $T_{g}^{l}: A \rightarrow \mathrm{C}$ and $T_{g}^{r}: A \rightarrow \mathrm{C}$ by

$$
T_{g}^{l}(f)=g \times f\left(s_{0}\right), \quad T_{g}^{r}(f)=f \times g\left(s_{0}\right) .
$$

For each $g \in A$ fix two regular measures $\mu_{g}^{l}$ and $\mu_{g}^{r}$ on $\partial A$ such that

$$
\begin{gathered}
\mu_{g}^{l}(f)=T_{g}^{l}(f), \quad \operatorname{var}\left(\mu_{g}^{l}\right)=\left\|T_{g}^{l}\right\|, \\
\mu_{g}^{r}(f)=T_{g}^{r}(f), \quad \operatorname{var}\left(\mu_{g}^{r}\right)=\left\|T_{g}^{r}\right\| \quad \text { for all } f \text { in } A .
\end{gathered}
$$

Inequality (8) shows that

$$
\left|\mu_{f_{\alpha}}^{l}\left(f_{\beta}\right)-1\right| \leqslant \sqrt{\varepsilon} \text { for any } \alpha \text { and all } \beta .
$$

By the definition of $\left(f_{\alpha}\right)$ we get

$$
\left|\mu_{f_{\alpha}}^{\prime}\left(\left\{s_{0}\right\}\right)-1\right| \leqslant \sqrt{\varepsilon} .
$$

Hence, because $\operatorname{var}\left(\mu_{f_{\alpha}}^{l}\right)=\left\|T_{f_{\alpha}}^{l}\right\|=1+\varepsilon$, the measure $\mu_{f_{\alpha}}^{l}$ is of the form

$$
\mu_{f_{\alpha}}^{l}=\delta_{s_{0}}+\Delta \mu_{f_{\alpha}}^{l}
$$

where $\delta_{s_{0}}$ is a Dirac measure concentrated at the point $s_{0}$ and $\operatorname{var}\left(\Delta \mu_{f_{\alpha}}^{l}\right) \leqslant 3 \sqrt{\varepsilon}$.

Now let $g_{0}$ be any element of $A$ such that $\left\|g_{0}\right\|=1=g_{0}\left(s_{0}\right)$. By (10) we get

$$
\begin{aligned}
\mu_{g_{0}}^{r}\left(f_{\alpha}\right) & =f_{\alpha} \times g_{0}\left(s_{0}\right)=\mu_{f_{\alpha}}^{l}\left(g_{0}\right) \\
& =g_{0}\left(s_{0}\right)+\Delta \mu_{f_{\alpha}}^{l}\left(g_{0}\right)=1+\Delta \mu_{f_{\alpha}}^{l}\left(g_{0}\right) .
\end{aligned}
$$

Hence

$$
\left|\mu_{g}^{r}\left(f_{\alpha}\right)-1\right| \leqslant 3 \sqrt{\varepsilon}
$$


In the same way as previously, we get

$$
\mu_{g_{0}}^{r}=\delta_{s_{0}}+\Delta \mu_{g_{0}}^{r} \quad \text { where } \operatorname{var}\left(\Delta \mu_{g_{0}}^{r}\right) \leqslant 7 \sqrt{\varepsilon} .
$$

Using this we can estimate the norm of $g_{0} \times g_{0}$ from below.

$$
\begin{aligned}
\left\|g_{0} \times g_{0}\right\| & \geqslant\left|g_{0} \times g_{0}\left(s_{0}\right)\right|=\left|\mu_{g_{0}}^{r}\left(g_{0}\right)\right|=\left|1+\Delta \mu_{g_{0}}^{r}\left(g_{0}\right)\right| \\
& \geqslant 1-7 \sqrt{\varepsilon} .
\end{aligned}
$$

Because $s_{0}$ is an arbitrary point of $\mathrm{Ch} A$ this proves that

$$
\|g \times g\| \geqslant(1-7 \sqrt{\varepsilon})\|g\|^{2} \text { for any } g \text { in } A \text {. }
$$

As an immediate consequence of (12) we conclude that the spectral radius of any element $g$ of the algebra $(A, \times)$ is not less than $(1-7 \sqrt{\varepsilon})\|g\|$. Hence by a theorem of Hirschfeld and Żelazko [1] one obtains the commutativity of the multiplication $X$ if $1-7 \sqrt{\varepsilon}>0$.

Applying (12) for $g=f_{\alpha}$ and using the commutativity of $\times$ we get that there exists a linear and $\times$-multiplicative functional $F_{\alpha}$ such that $\left|F_{\alpha}\left(f_{\alpha}\right)\right| \geqslant 1-7 \sqrt{\varepsilon}$. For any $f$ in $A$ of norm equal one we have

$$
(1+\varepsilon)\left\|F_{\alpha}\right\| \geqslant\left\|F_{\alpha}\right\|\|f \times f\| \geqslant\left|F_{\alpha}(f \times f)\right|=\left|F_{\alpha}(f)\right|^{2},
$$

hence

$$
(1+\varepsilon)\left\|F_{\alpha}\right\| \geqslant\left\|F_{\alpha}\right\|^{2}, \quad \text { so }\left\|F_{\alpha}\right\| \leqslant 1+\varepsilon
$$

Let $\nu_{\alpha}$ be a regular measure on $\partial A$ which represents the functional $F_{\alpha}$ and such that $\operatorname{var}\left(\nu_{\alpha}\right)=\left\|F_{\alpha}\right\|$. We have

$$
\left\{\begin{array}{l}
\left|\nu_{\alpha}\left(f_{\alpha}\right)\right| \geqslant 1-7 \sqrt{\varepsilon} \\
\operatorname{var}\left(\nu_{\alpha}\right) \leqslant 1+\varepsilon \\
\nu_{\alpha}(1)=1 \text { for all indices } \alpha
\end{array}\right.
$$

Taking a net finer than $\left(f_{\alpha}\right)$ and using the weak $*$ compactness of $\partial A$ we can assume, without loss of generality, that the net $\left(\nu_{\alpha}\right)$ is weak $*$ convergent to the measure $\nu_{0}$. The measure $\nu_{0}$ also represents a linear and $\times$-multiplicative functional $F_{0}$ on $A$. From (13) we derive that the measure $\nu_{0}$ is of the form

$$
\nu_{0}=\delta_{s_{0}}+\Delta \nu_{s_{0}} \quad \text { where } \operatorname{var}\left(\Delta \nu_{s_{0}}\right) \leqslant c_{1} \sqrt{\varepsilon} .
$$

From (14) for any $f, g$ in $A$ we find

$$
\begin{aligned}
f \times g\left(s_{0}\right) & +\Delta \nu_{s_{0}}(f \times g)=\nu_{0}(f \times g)=\nu_{0}(f) \cdot \nu_{0}(g) \\
& =\left(f\left(s_{0}\right)+\Delta \nu_{s_{0}}(f)\right) \cdot\left(g\left(s_{0}\right)+\Delta \nu_{s_{0}}(g)\right) \\
& =f\left(s_{0}\right) \cdot g\left(s_{0}\right)+\Delta \nu_{s_{0}}(f) \cdot g\left(s_{0}\right)+f\left(s_{0}\right) \cdot \Delta \nu_{s_{0}}(g)+\Delta \nu_{s_{0}}(f) \cdot \Delta \nu_{s_{0}}\left(g_{0}\right) .
\end{aligned}
$$

Hence

$$
\begin{aligned}
\left|f \times g\left(s_{0}\right)-f \cdot g\left(s_{0}\right)\right| & \leqslant \operatorname{var}\left(\Delta v_{s_{0}}\right)\left(2\|f\|\|g\|+\operatorname{var}\left(\Delta \nu_{s_{0}}\right) \cdot\|f\|\|g\|\right) \\
& \leqslant c_{1} \sqrt{\varepsilon}\left(2+c_{1} \sqrt{\varepsilon}\right) \cdot\|f\|\|g\|=c \sqrt{\varepsilon}\|f\|\|g\| .
\end{aligned}
$$


Because $s_{0}$ is an arbitrary point in $\mathrm{Ch} A$, which is a dense subset of $\partial A$, the above statement proves (iii) and ends the proof of the theorem.

Corollary 1. Suppose $(A, \cdot)$ is a complex function algebra. Let $\times$ be any associative multiplication on the Banach space $A$ with the same unit and such that $(A, \times)$ is a Banach algebra (this means such that $\|f \times g\| \leqslant\|f\|\|g\|$ for all $f, g$ in $A$ ). Then the new multiplication $\times$ and the original one coincide.

The above corollary can be also formulated in the following way, giving a generalization of Nagasawa's Theorem.

Corollary 2. Let $(A, \cdot)$ be a complex function algebra with unit $\mathbf{1}_{A}$ and let $B$ be any Banach algebra with unit $\mathbf{1}_{B}$. Suppose $T$ is a linear isometry from $A$ onto $B$ such that $T \mathbf{1}_{A}=\mathbf{1}_{B}$. Then $T$ is an algebra isomorphism of $A$ and $B$.

Notice that we have only considered complex Banach algebras. The theorem and the corollaries are not valid for real function algebras, even in two dimensions. To prove this let $A=\left(\mathbf{R}^{2}, \cdot\|\cdot\|_{\infty}\right)$ be the two dimensional real function algebra and let $\rho_{t}: \mathbf{R}^{2} \times \mathbf{R}^{2} \rightarrow \mathbf{R}^{2}$.

$$
\rho_{t}\left((x, y),\left(x^{\prime}, y^{\prime}\right)\right)=\left(x x^{\prime}-t(x-y)\left(x^{\prime}-y^{\prime}\right), y y^{\prime}-t(x-y)\left(x^{\prime}-y^{\prime}\right)\right) .
$$

A direct computation shows that for any $0 \leqslant t \leqslant 1 / 2$ the bilinear map $\rho_{t}$ is a commutative, associative multiplication on $\mathbf{R}^{2}$ such that $\left\|\rho_{t}\right\|=1$ and

$$
\rho_{t}((1,1),(x, y))=(x, y) \text { for any }(x, y) \in \mathbf{R}^{2} .
$$

Let us end the paper with a natural problem arising from Corollary 1.

Problem. Characterize those Banach spaces $A$ and elements $e$ which admit a unique multiplication $X$ on $A$ so that $(A, \times)$ is a Banach algebra with unit $e$.

\section{REFERENCES}

1. R. A. Hirschfeld and W. Żelazko, On spectral norm Banach algebras, Bull. Acad. Polon. Sci. (3) 16 (1968), 195-199.

2. B. E. Johnson, Perturbations of Banach algebras, Proc. London Math. Soc. (3) 34 (1977), 439-458.

3. R. V. Kadison and D. Kastler, Perturbations of von Neumann algebras I, stability of type, Amer. J. Math. 94 (1972), 38-54.

4. J. Philips, Perturbations of $C^{*}$-algebras, Indiana Univ. Math. J. 23 (1973-4), 1167-1176.

5. R. Rochberg, Deformations of uniform algebras, Proc. London Math. Soc. 39 (3) (1979), 93-118.

Institute of Mathematics, Warsaw University, Warsaw, POland 